\title{
NOTICES
}

\section{Observing Time on Kitt Peak}

$\mathrm{T}$ HE Kitt Peak National Observatory was established to provide American astronomers and qualified graduate students with modern telescopic and instrumental equipment for astronomical research. Also, the Board of Directors of AURA (Association of Universities for Research in Astronomy) has set the policy that about $60 \%$ of the observing time on Kitt Peak shall be made available to visiting astronomers and graduate students.

No charge is made for telescope time, but visiting astronomers will be billed for food and lodging while working on Kitt Peak.

At the present time, two moderate-sized, Cassegrain focus telescopes are in operation on Kitt Peak: a 16-inch and a 36-inch. Auxiliary equipment consists of photoelectric photometers for both telescopes, and a grating spectrograph for the 36-inch.

Since a number of inquiries and requests for observing time have already been received, an Observers' Information Leaflet and an Observing Time Request Form have been prepared for those persons who are interested in using the Kitt Peak facilities. Copies may be obtained by writing to: Office of the Director, Kitt Peak National Observatory, 950 North Cherry Avenue, Tucson, Arizona.

It is AURA policy that observing-time requests be granted on the basis of scientific merit of the proposed program. In most cases, the Observatory staff reviews proposals and makes recommendations regarding assignments; occasionally, the advice of outside referees may be sought. Visiting astronomers are expected to do their own observing on Kitt Peak, although they may bring a qualified assistant if their program requires it.

Subject to existing Federal regulations, qualified foreign astronomers may use the Observatory's facilities.
In cases of U.S. and foreign programs judged to be of equal merit, however, preference will be given to astronomers resident in the U.S., because of the national character of the Observatory.

To the extent that funds are available, the Observatory's graduate student program pays for travel and subsistence expenses of qualified, advanced-degree U.S. students.

Although the resident staff wishes to be as helpful as possible to visitors, its responsibility is limited to giving advice and initial assistance for the efficient use of the instruments. The Observatory staff and assistants will not do observing for visitors except under emergency conditions.

In addition to the telescopes on Kitt Peak, there are at the Tucson headquarters a number of laboratory instruments, such as an electronic digital computer, a Hilger microphotometer, a Cary monochromator and spectrometer, measuring machines for direct photographs and spectrograms, a spectrocomparator, and binocular microscope viewers. The main Observatory library, electronics and photographic laboratories, and the instrument and optical shops are also located in the Tucson headquarters buildings.

Depending upon availability of office space, astronomers on sabbatical or other long-term leave may work at the Observatory while in residence in Tucson.

The Observatory staff welcomes inquiries from those astronomers and graduate students who have research programs that may merit the use of the Kitt Peak National Observatory facilities.

\author{
N. U. Mayall \\ Observatory Director \\ Kitt Peak National Observatory, \\ Tucson, Arizona
}

